\title{
Serum MicroRNA-371a-3p Levels Predict Viable Germ Cell Tumor in Chemotherapy-naïve Patients Undergoing Retroperitoneal Lymph Node Dissection
}

\author{
John T. Lafin ${ }^{a, 1}$, Nirmish Singla ${ }^{a, 1}$, Solomon L. Woldua ${ }^{a}$, Yair Lotan ${ }^{a}$, Cheryl M. Lewis $^{b}$, \\ Kuntal Majmudar $^{b}$, Anna Savelyeva ${ }^{\mathrm{a}}$, Payal Kapur ${ }^{\mathrm{b}}$, Vitaly Margulis ${ }^{\mathrm{a}, \mathrm{c}}$, Douglas W. Strand ${ }^{\mathrm{a}}$, \\ Matthew J. Murrayd,e, James F. Amatruda ${ }^{f}$, Aditya Bagrodiaa, ${ }^{\star}$ \\ aDepartment of Urology, University of Texas Southwestern Medical Center, Dallas, TX, USA \\ ${ }^{b}$ Department of Pathology, University of Texas Southwestern Medical Center, Dallas, TX, USA \\ 'Department of Urology, I.M. Sechenov First Moscow State University, Moscow, Russia \\ dDepartment of Pathology, University of Cambridge, Cambridge, UK \\ eDepartment of Pediatric Hematology and Oncology, Cambridge University Hospitals NHS \\ Foundation Trust, Cambridge, UK \\ fDepartment of Pediatrics, University of Texas Southwestern Medical Center, Dallas, TX, USA
}

\begin{abstract}
Serum-based biomarkers ( $\alpha$-fetoprotein, $\beta$-human chorionic gonadotropin, and lactate dehydrogenase) are integral to the diagnosis, management, and follow-up of patients with testicular germ cell tumors (GCTs). The clinical utility of these markers is tempered by limited sensitivity and specificity, as they are expressed in only a subset of GCTs and levels may be normal in the setting of early recurrence or low tumor burden (marker-negative, radiographically occult metastases). Serum microRNAs (miRNAs; miRs)—small noncoding RNAs involved in epigenetic regulation of gene expression - are emerging as candidate biomarkers for diagnosing and monitoring viable GCT, regardless of histology [1,2]. The capacity of miRNAs to predict viable GCT in chemotherapy-naïve patients has not been explored. The ability to accurately personalize the decision to forego or pursue primary treatment (retroperitoneal lymph node dissection [RPLND], chemotherapy, or radiotherapy) in a cohort of patients arguably best suited for surveillance would mitigate the morbidity associated with overtreatment. We evaluated the performance characteristics of serum miRNA levels in predicting viable GCT among chemotherapy-naïve patients undergoing primary RPLND.
\end{abstract}

We prospectively collected serum samples from consecutive chemotherapy-naïve patients with type II GCT undergoing primary RPLND at our institution between 2016 and 2019

\footnotetext{
*Corresponding author. Department of Urology, University of Texas, Southwestern Medical Center, 2001 Inwood Road, Dallas, TX 75390-9110, USA. Tel. +1 214 6458765; Fax: +1 214 6488676. aditya.bagrodia@utsouthwestern.edu (A. Bagrodia).

${ }^{1}$ Joint first authors.

Conflicts of interest: The authors have nothing to disclose.

Appendix A. Supplementary data

Supplementary material related to this article can be found, in the online version, at doi:https://doi.org/10.1016/j.eururo.2019.10.005.
} 
immediately before surgery. Preoperative cross-sectional staging imaging and conventional serum tumor marker (STM) measurements were carried out within $10 \mathrm{~d}$ of surgery. All patients had either clinical stage I or II GCT with no radiographic evidence of distant metastases. Bilateral full-template or extended modified template (extending to the contralateral ureter above the inferior mesenteric artery) nerve-sparing RPLND was performed. RNA extraction, initial quality control and serum miRNA quantification were carried out as described by Murray et al. [3]. RPLND histology was classified as benign, viable GCT, or teratoma only. Corresponding performance characteristics (area under the curve $[\mathrm{AUC}]$ analysis of receiver operator characteristic [ROC] curves, accuracy, sensitivity, specificity, positive predictive value [PPV], and negative predictive value [NPV]) were calculated for each miRNA signature.

Our cohort consisted of 24 patients with clinical stage I (50\%) or II (50\%) GCT undergoing primary RPLND. Orchiectomy histology was pure seminoma in four (16.7\%), nonseminoma GCT (NSGCT) in 18 (75\%), and burned-out tumor in two patients (8.3\%) with clinical stage II disease. Final RPLND histology was benign in ten (41.7\%), pure teratoma in three $(12.5 \%)$, and viable GCT in 11 patients (45.8\%). Seminoma and teratoma were identified on RPLND for patients with burned-out primary tumor. The smallest foci of viable GCT identified were, in some cases, $<5 \mathrm{~mm}$. Four patients (16.7\%) received chemotherapy after RPLND, including one patient who experienced recurrence, one patient with limbic encephalitis paraneoplastic syndrome, and two patients with N2 NSGCT who received adjuvant chemotherapy. Out of the prototypical four-member putative serum miRNA panel for detection of GCT, miR-371a-3p has consistently been the most sensitive [2,4]. We also included miR-375 in our panel because of a recent report indicating high expression in teratoma tissue [5]. To determine if serum miR-371a-3p predicts the presence of viable GCT at RPLND, patients were divided into three groups on the basis of RPLND pathology: benign, pure teratoma, or viable GCT. miR-371a-3p was the most discriminatory serum miRNA for viable GCT, exhibiting 13 000-fold greater expression over teratoma or benign pathology. On ROC analysis, miR-371a-3p had an AUC of 0.965 , sensitivity of $100 \%$ and specificity of $92 \%$. The AUC for other serum miRNAs in predicting viable GCT was 0.874 for miR-367-3p, 0.846 for miR-372-3p, and 0.720 for miR-373-3p. The performance of miR-371a-3p did not differ significantly from the full panel $(p>0.05)$. These serum miRNAs, including miR-375, were not predictive of pure teratoma.

In this prospective study, we found that miR-371a-3p exhibited striking performance characteristics for accurate discrimination of viable GCT from benign processes and pure teratoma in patients undergoing primary RPLND with normal STMs. miR-371a-3p yielded an AUC of 0.965 , sensitivity of $100 \%$ and specificity of $92 \%$ at a threshold of twofold over normal serum (Fig. 1). miR-371a-3p was elevated for both nonteratomatous NSGCT and pure seminoma, despite normal conventional STMs. These novel results demonstrate strong performance characteristics for miR-371a-3p for detection of occult or low-volume retroperitoneal disease. Despite being limited by small numbers and inability to sub stratify, if validated in larger cohorts these data could suggest a basis for revolutionizing how patients with early-stage GCT are treated. No other studies have described the performance characteristics of miRNAs with pathologic confirmation of the presence or absence of smallvolume metastatic disease in the context of negative STMs. Two large clinical trials 
(AGCT1531 [NCT03067181], which is accruing; and SWOG-S1823, which is expected to open soon) are further studying the role of miRNAs in patients with early-stage disease. In the largest prospective study to evaluate the performance of miR-371a-3p as a biomarker in GCT, Dieckmann et al [2] observed 90\% sensitivity, 94\% specificity, 97\% PPV, 83\% NPV, and an AUC of 0.966 for primary diagnosis of malignant GCT, similar to the performance characteristics reported here. Our study is unique in that we were able to pathologically verify the presence or absence of viable GCTs before clinical relapse.

Our data suggest excellent performance characteristics of miRNAs, particularly miR-371a-3p, for accurate differentiation of small-volume viable GCT from benign processes or pure teratoma in patients undergoing primary RPLND, with an AUC of 0.965 and $>90 \%$ sensitivity and specificity. Although none of these miRNAs accurately predicted teratoma, our data set was small and evaluation of miR-375 is still in the early stages. If validated in larger cohorts, these data suggest a basis for implementing precision medicine strategies for patients with early-stage GCTs. Owing to the early stage of this work and the technical expertise required, current clinical decision-making should not rely on miRNA assay results.

\section{Supplementary Material}

Refer to Web version on PubMed Central for supplementary material.

\section{Acknowledgments:}

The authors would like to thank Aphrihl Dennis and the University of Texas Southwestern Tissue Repository for acquisition and storage of patient serum samples. This work was supported by a Ruth L. Kirschstein National Research Service Award (T32 CA136515-09; N.S.), the University of Texas Southwestern Medical Center Physician Scientist Training Program (N.S.), a National Cancer Institute of the National Institutes of Health award (5 P30 CA142543 09; C.L. and K.M), a St. Baldrick's Consortium award (358099; M.J.M. and J.FA.), grant RP170152 from the Cancer Prevention and Research Institute of Texas (A.B. and J.F. A.), the Malignant Germ Cell International Consortium (M.J.M., J.F.A., and A.B.) and a Dedman Family Scholarship in Clinical Care (A.B).

\section{References}

[1]. Murray MJ, Halsall DJ, Hook CE, Williams DM, Nicholson JC, Coleman N. Identification of microRNAs from the miR-371 373 and miR-302 clusters as potential serum biomarkers of malignant germ cell tumors. Am J Clin Pathol 2011;135:119-25. [PubMed: 21173133]

[2]. Dieckmann KP, Radtke A, Geczi L, et al. Serum levels of microRNA-371a-3p (M371 test) as a new biomarker of testicular germ cell tumors: results of a prospective multicentric study. J Clin Oncol 2019;37(16):1412-23, JCO1801480, Jun 1,. [PubMed: 30875280]

[3]. Murray MJ, Bell E, Raby KL, et al. A pipeline to quantify serum and cerebrospinal fluid microRNAs for diagnosis and detection of relapse in paediatric malignant germ-cell tumours. $\mathrm{Br}$ J Cancer 2016;114(2):151-62. [PubMed: 26671749]

[4]. Leao R, van Agthoven T, Figueiredo A, et al. Serum miRNA predicts viable disease after chemotherapy in patients with testicular nonseminoma germ cell tumor. J Urol 2018;200(1):12635. [PubMed: 29474847]

[5]. Shen H, Shih J, Hollern DP, et al. Integrated molecular characterization of testicular germ cell tumors. Cell Rep 2018;23:3392-406. [PubMed: 29898407] 

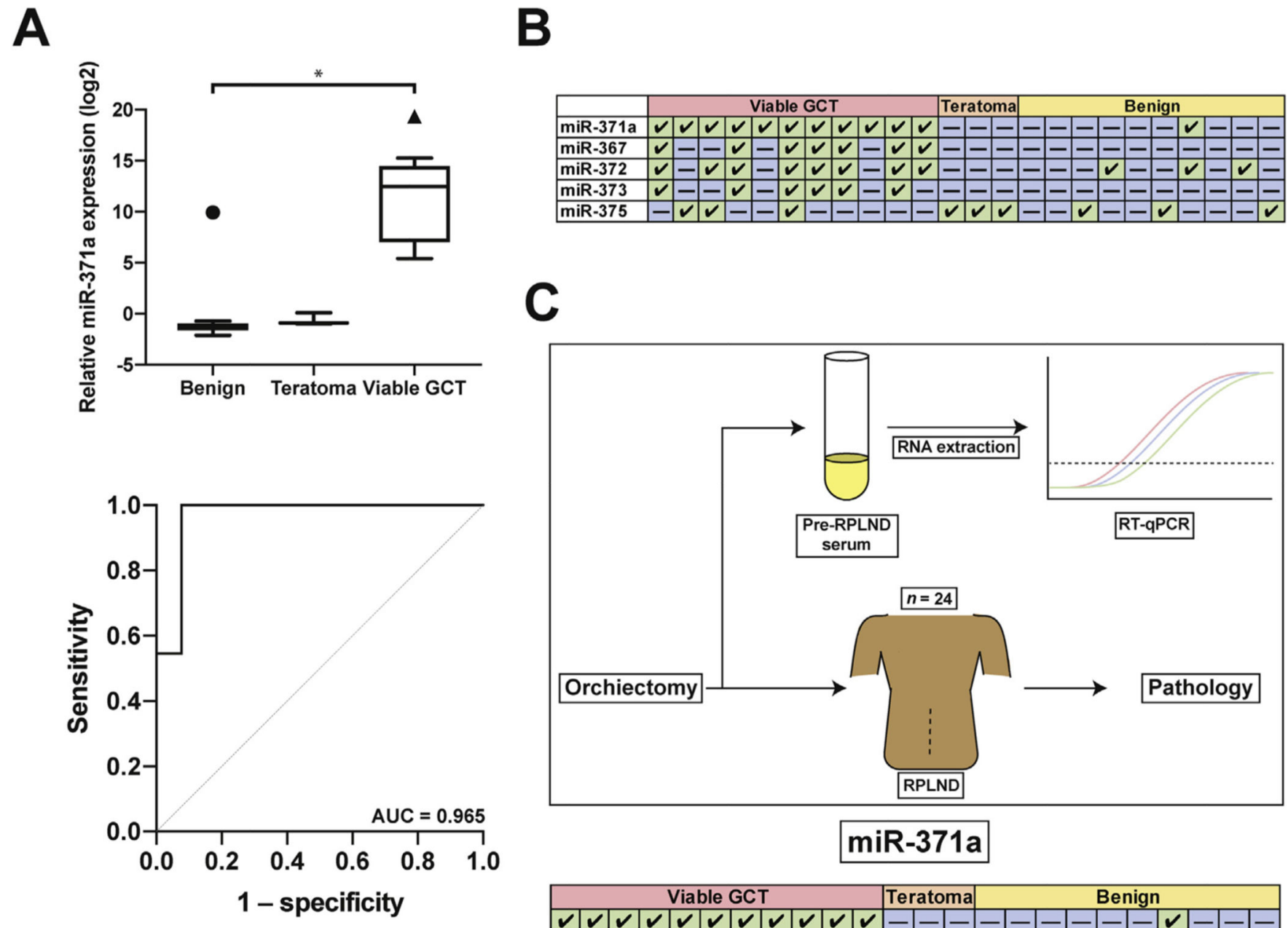

miR-371a

\begin{tabular}{|c|c|}
\hline Viable GCT & Teratoma \\
\hline 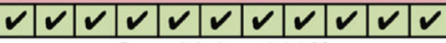 & $|-|-|-|-|-|-|-|-|-| v|-|-\mid-$ \\
\hline Sensitivity: $100 \%$ & Specificity: $\mathbf{9 2} \%$ \\
\hline
\end{tabular}

Fig. 1 -.

(A) Top: Serum miR-371a-3p expression relative to normal serum. Symbols represent outliers as determined by Tukey's method. GCT=germ cell tumor. Bottom: Receiver operating characteristic curve for miR-371a-3p. AUC=area under the curve. (B) Summary table of results. Green box with a check indicates a positive result (relative expression over threshold); blue box with a dash indicates a negative result. Thresholds and performance metrics are outlined in Supplementary Table 1. (C) Graphical summary. Serum was taken immediately before retroperitoneal lymph node dissection (RPLND) for RNA extraction and reverse transcription and qualitative polymerase chain reaction (RT-qPCR). Results were compared to RPLND pathology. miR-371a-3p summary results and performance metrics are reported. 